\title{
Automorphisms of metacyclic groups
}

\author{
Haimiao Chen * \\ Department of Mathematics, Beijing Technology and Business University, Beijing, China \\ Yueshan Xiong ${ }^{\dagger}$ \\ School of Mathematics and Statics, Huazhong University of Science and Technology, Wuhan, China \\ Zhongjian Zhu ${ }^{\ddagger}$ \\ School of Mathematics and Information Science, Wenzhou University, Wenzhou, China
}

\begin{abstract}
A metacyclic group $H$ can be presented as $\left\langle\alpha, \beta \mid \alpha^{n}=1, \beta^{m}=\alpha^{t}, \beta \alpha \beta^{-1}=\alpha^{r}\right\rangle$ for some $n, m, t, r$. Each endomorphism $\sigma$ of $H$ is determined by $\sigma(\alpha)=\alpha^{x_{1}} \beta^{y_{1}}, \sigma(\beta)=\alpha^{x_{2}} \beta^{y_{2}}$ for some integers $x_{1}, x_{2}, y_{1}, y_{2}$. We give sufficient and necessary conditions on $x_{1}, x_{2}, y_{1}, y_{2}$ for $\sigma$ to be an automorphism.
\end{abstract}

Keywords: automorphism, metacyclic group, linear congruence equation. MSC 2010: 20D45.

\section{Introduction}

A finite group $G$ is metacyclic if it contains a cyclic, normal subgroup $N$ such that $G / N$ is also cyclic. In some sense, metacyclic groups can be regarded as the simplest ones other than abelian groups.

As a natural object, the automorphism group of a metacyclic group has been widely studied. In 1970, Davitt [5] showed that if $G$ is a metacyclic $p$-group with $p \neq 2$, then the order $G$ divides that of $\operatorname{Aut}(G)$. In 2006, Bidwell and Curran [1] found the order and the structure of Aut $(G)$ when $G$ is a split metacyclic $p$-group with $p \neq 2$, and in 2007, Curran 3 obtained similar results for split metacyclic 2-groups. In 2008, Curran [4] determined $\operatorname{Aut}(G)$ when $G$ is a nonsplit metacyclic $p$-group with $p \neq 2$. In 2009, Golasiński and Gonçalves [6] determined $\operatorname{Aut}(G)$ for any split metacyclic group $G$. The case of nonsplit metacyclic 2-groups remains unknown.

In this paper we aim at writing down all of the automorphisms for a general metacyclic group. One of our main motivations stems from the study of regular Cayley maps on metacyclic groups (see [2]), which requires an explicit formula for a general automorphism.

\footnotetext{
*Email: chenhm@math.pku.edu.cn

${ }^{\dagger}$ Email: xiongyueshan@gmail.com

‡Email: zhuzhongjianzzj@126.com
} 
It is well-known (see Section 3.7 of [8]) that each metacyclic group can be presented as

$$
\left\langle\alpha, \beta \mid \alpha^{n}=1, \beta^{m}=\alpha^{t}, \beta \alpha \beta^{-1}=\alpha^{r}\right\rangle
$$

for some positive integers $n, m, r, t$ satisfying

$$
r^{m}-1 \equiv t(r-1) \equiv 0 \quad(\bmod n) .
$$

Denote this group by $H=H(n, m ; t, r)$. There is an extension

$$
1 \rightarrow \mathbb{Z} / n \mathbb{Z} \rightarrow H \rightarrow \mathbb{Z} / m \mathbb{Z} \rightarrow 1
$$

where $\mathbb{Z} / n \mathbb{Z} \cong\langle\alpha\rangle \triangleleft H$ and $\mathbb{Z} / m \mathbb{Z} \cong H /\langle\alpha\rangle$. It may happen that two groups given by different values of $n, m, t, r$ are isomorphic. A complete classification (up to isomorphism) for finite metacyclic groups was obtained by Hempel [7] in 2000.

In the presentation (1), we may assume $t \mid n$ which we do from now on. To see this, choose $u, v$ such that $u n+v t=(n, t)$, then $(v, n /(n, t))=1$. Let $w$ be the product of all prime factors of $m$ that do not divide $v$ and let $v^{\prime}=v+w n /(n, t)$, then $\left(v^{\prime}, m\right)=1$. Replacing $\beta$ by $\check{\beta}=\beta^{v^{\prime}}$, we get another presentation: $H=\left\langle\alpha, \check{\beta} \mid \alpha^{n}=1, \check{\beta}^{m}=\alpha^{(n, t)}, \check{\beta} \alpha \check{\beta}^{-1}=\alpha^{r^{v^{\prime}}}\right\rangle$.

Obviously each element can be written as $\alpha^{u} \beta^{v}$; note that $\alpha^{u} \beta^{v}=1$ if and only if $m \mid v$ and $n \mid(u+t v / m)$. Each endomorphism $\sigma$ of $H$ is determined by $\sigma(\alpha)=\alpha^{x_{1}} \beta^{y_{1}}, \sigma(\beta)=\alpha^{x_{2}} \beta^{y_{2}}$ for some integers $x_{1}, x_{2}, y_{1}, y_{2}$. The main result of this paper gives sufficient and necessary conditions on $x_{1}, x_{2}, y_{1}, y_{2}$, for $\sigma$ to be an automorphism. They consist of two parts, ensuring $\sigma$ to be invertible and well-defined, respectively. Skillfully using elementary number theoretic techniques, we manage to reduce the second part to linear congruence equations. It turns out that the situation concerning the prime 2 is quite subtle, and this reflects the difficulty in determining the automorphism groups of nonsplit metacyclic 2-groups.

\section{Notation and Convention}

- For an integer $N>0$, denote $\mathbb{Z} / N \mathbb{Z}$ by $\mathbb{Z}_{N}$ and regard it as a quotient ring of $\mathbb{Z}$. For $u \in \mathbb{Z}$, denote its image under the quotient $\mathbb{Z} \rightarrow \mathbb{Z}_{N}$ also by $u$.

- Given integers $u, s$ with $u>0$, set $[u]_{s}=1+s+\cdots+s^{u-1}$, so that $(s-1)[u]_{s}=s^{u}-1$; for a prime number $p$, let $\operatorname{deg}_{p}(u)$ denote the largest integer $s$ with $p^{s} \mid u$.

- Denote $\alpha^{u}$ by $\exp _{\alpha}(u)$ when the expression for $u$ is too long.

- To avoid subtleties, we assume $x_{1}, x_{2}, y_{1}, y_{2}$ to be positive, and usually write an element of $H$ as $\alpha^{u} \beta^{v}$ with $u, v>0$.

\section{Determining all automorphisms}

\subsection{Preparation}

Lemma 2.1. If $s>1$ with $\operatorname{deg}_{p}(s-1)=\ell \geq 1$ and $x>0$ with $\operatorname{deg}_{p}(x)=u \geq 0$, then 
(I) $[x]_{s} \equiv\left\{\begin{array}{ll}x, & p \neq 2 \text { or } u=0, \\ \left(1+2^{\ell-1}\right) x, & p=2 \text { and } u>0\end{array}\left(\bmod p^{\ell+u}\right)\right.$;

(II) $s^{x}-1 \equiv\left\{\begin{array}{ll}(s-1) x, & p \neq 2 \text { or } u=0, \\ \left(s-1+2^{2 \ell-1}\right) x, & p=2 \text { and } u>0\end{array}\left(\bmod p^{2 \ell+u}\right)\right.$.

Proof. We only prove (I), then (II) follows from the identity $(s-1)[x]_{s}=s^{x}-1$.

If $u=0$, then $s \equiv 1\left(\bmod p^{\ell+u}\right)$, so $[x]_{s} \equiv x\left(\bmod p^{\ell+u}\right)$.

Let us assume $u>0$. Write $s=1+p^{\ell} h$ with $p \nmid h$. Note that

$$
\begin{aligned}
\operatorname{deg}_{p}\left(\left(\begin{array}{c}
p^{u} \\
j
\end{array}\right)\right) & =\operatorname{deg}_{p}\left(\frac{\left(p^{u}\right) !}{j !\left(p^{u}-j\right) !}\right)=\sum_{i=0}^{j-1} \operatorname{deg}_{p}\left(p^{u}-i\right)-\sum_{i=1}^{j} \operatorname{deg}_{p}(i) \\
& =u-\operatorname{deg}_{p}(j)+\sum_{i=1}^{j-1}\left(\operatorname{deg}_{p}\left(p^{u}-i\right)-\operatorname{deg}_{p}(i)\right) \\
& =u-\operatorname{deg}_{p}(j) .
\end{aligned}
$$

If $p \neq 2$, then

$$
\left[p^{u}\right]_{s}=\sum_{i=0}^{p^{u}-1}\left(1+p^{\ell} h\right)^{i}=\sum_{i=0}^{p^{u}-1} \sum_{j=0}^{i}\left(\begin{array}{l}
i \\
j
\end{array}\right)\left(p^{\ell} h\right)^{j}=\sum_{j=1}^{p^{u}}\left(\begin{array}{c}
p^{u} \\
j
\end{array}\right)\left(p^{\ell} h\right)^{j-1} \equiv p^{u} \quad\left(\bmod p^{\ell+u}\right),
$$

using that for all $j \geq 2$,

$$
\operatorname{deg}_{p}\left(\left(\begin{array}{c}
p^{u} \\
j
\end{array}\right)\right)=u-\operatorname{deg}_{p}(j) \geq u-(j-2) \ell=(\ell+u)-(j-1) \ell .
$$

Hence $s^{p^{u}}=(s-1)\left[p^{u}\right]_{s}+1 \equiv 1\left(\bmod p^{\ell+u}\right)$. Writing $x=p^{u} x^{\prime}$ with $p \nmid x^{\prime}$, we have

$$
[x]_{s}=\left[p^{u}\right]_{s} \cdot \sum_{j=0}^{x^{\prime}-1}\left(s^{p^{u}}\right)^{j} \equiv x^{\prime}\left[p^{u}\right]_{s} \equiv x \quad\left(\bmod p^{\ell+u}\right) .
$$

If $p=2$, then using that for all $j \geq 3$,

$$
\operatorname{deg}_{2}\left(\left(\begin{array}{c}
2^{u} \\
j
\end{array}\right)\right)=u-\operatorname{deg}_{2}(j) \geq u-(j-2) \ell=(\ell+u)-(j-1) \ell,
$$

we obtain

$$
\left[2^{u}\right]_{s}=\sum_{j=1}^{2^{u}}\left(\begin{array}{c}
2^{u} \\
j
\end{array}\right)\left(2^{\ell} h\right)^{j-1} \equiv 2^{u}+\left(\begin{array}{c}
2^{u} \\
2
\end{array}\right) 2^{\ell} h \equiv 2^{u}\left(1+2^{\ell-1}\right) \quad\left(\bmod 2^{\ell+u}\right) .
$$

Hence $s^{2^{u}}=(s-1)\left[2^{u}\right]_{s}+1 \equiv 1\left(\bmod 2^{\ell+u}\right)$. Writing $x=2^{u} x^{\prime}$ with $2 \nmid x^{\prime}$, we have

$$
[x]_{s}=\left[2^{u}\right]_{s} \cdot \sum_{j=0}^{x^{\prime}-1}\left(s^{2^{u}}\right)^{j} \equiv x^{\prime}\left[2^{u}\right]_{s} \equiv\left(1+2^{\ell-1}\right) x \quad\left(\bmod 2^{\ell+u}\right) .
$$




\subsection{The method}

It follows from (11) that, for $k, u, v, u^{\prime}, v^{\prime}>0$,

$$
\begin{aligned}
\beta^{v} \alpha^{u} & =\alpha^{u r^{v}} \beta^{v}, \\
\left(\alpha^{u} \beta^{v}\right)\left(\alpha^{u^{\prime}} \beta^{v^{\prime}}\right) & =\alpha^{u+u^{\prime} r^{v}} \beta^{v+v^{\prime}}, \\
\left(\alpha^{u} \beta^{v}\right)^{k} & =\alpha^{u[k]_{r} v} \beta^{v k}, \\
{\left[\alpha^{u} \beta^{v}, \alpha^{u^{\prime}} \beta^{v^{\prime}}\right] } & =\exp _{\alpha}\left(u^{\prime}\left(r^{v}-1\right)-u\left(r^{v^{\prime}}-1\right)\right),
\end{aligned}
$$

where the notation $[\theta, \eta]=\theta \eta \theta^{-1} \eta^{-1}$ for commutator is adopted.

In view of (6) , the commutator subgroup $[H, H]$ is generated by $\alpha^{r-1}$. The abelianization $H^{\text {ab }}:=H /[H, H]$ has a presentation

$$
\langle\bar{\alpha}, \bar{\beta} \mid q \bar{\alpha}=0, m \bar{\beta}=t \bar{\alpha}\rangle, \quad \text { with } \quad q=(r-1, n),
$$

where additive notations are used and $\bar{\alpha}+\bar{\beta}=\bar{\beta}+\bar{\alpha}$ is implicitly assumed.

Lemma 2.2. There exists a homomorphism $\sigma: H \rightarrow H$ with $\sigma(\alpha)=\alpha^{x_{1}} \beta^{y_{1}}, \sigma(\beta)=\alpha^{x_{2}} \beta^{y_{2}}$ if and only if

$$
\begin{aligned}
(r-1, t) y_{1} \equiv 0 & & (\bmod m), \\
x_{2}[m]_{r} y_{2}+t y_{2}-x_{1}[t]_{r^{y_{1}}}-\frac{t y_{1}}{m} t \equiv 0 & & (\bmod n), \\
x_{2}\left(r^{y_{1}}-1\right)+x_{1}\left([r]_{r^{y_{1}}}-r^{y_{2}}\right)+\frac{(r-1) y_{1}}{m} t \equiv 0 & & (\bmod n) .
\end{aligned}
$$

Proof. Sufficient and necessary conditions for $\sigma$ to be well-defined are:

$$
\begin{aligned}
\alpha^{x_{1}[n]_{r} y_{1}} \beta^{y_{1} n} & =\sigma(\alpha)^{n}=1, \\
\alpha^{x_{2}[m]_{r} y_{2}} \beta^{y_{2} m}=\sigma(\beta)^{m} & =\sigma(\alpha)^{t}=\alpha^{x_{1}[t]_{r} y_{1}} \beta^{y_{1} t}, \\
\alpha^{x_{2}} \beta^{y_{2}} \alpha^{x_{1}} \beta^{y_{1}} \beta^{-y_{2}} \alpha^{-x_{2}}=\sigma(\beta) \sigma(\alpha) \sigma(\beta)^{-1} & =\sigma(\alpha)^{r}=\alpha^{x_{1}[r]_{r} y_{1}} \beta^{y_{1} r} ;
\end{aligned}
$$

equivalently,

$$
\begin{array}{rrrr}
n y_{1} \equiv 0 & (\bmod m), & x_{1}[n]_{r^{y_{1}}}+\frac{n y_{1}}{m} t \equiv 0 & (\bmod n), \\
t y_{1} \equiv 0 & (\bmod m), & x_{2}[m]_{r} y_{2}+y_{2} t \equiv x_{1}[t]_{r^{y_{1}}}+\frac{t y_{1}}{m} t & (\bmod n), \\
(r-1) y_{1} \equiv 0 & (\bmod m), & x_{2}\left(1-r^{y_{1}}\right)+x_{1} r^{y_{2}} \equiv x_{1}[r]_{r} y_{1}+\frac{(r-1) y_{1}}{m} t & (\bmod n) .
\end{array}
$$

Due to $t \mid n$, the first halves of (11), (12), (13) are equivalent to the single condition (8). Then the second half of (11) can be omitted: for each prime divisor $p$ of $n$, if $p \mid r^{y_{1}}-1$, then by Lemma 2.1 (I), $\operatorname{deg}_{p}\left([n]_{r} y_{1}\right) \geq \operatorname{deg}_{p}(n)$; if $p \nmid r^{y_{1}}-1$, then since $r^{n y_{1}}-1$ is a multiple of $r^{m}-1$, we also have $\operatorname{deg}_{p}\left([n]_{r^{y_{1}}}\right)=\operatorname{deg}_{p}\left(r^{n y_{1}}-1\right) \geq \operatorname{deg}_{p}\left(r^{m}-1\right) \geq \operatorname{deg}_{p}(n)$. 
Let $\Lambda$ denote the set of prime divisors of $n m$, and for each $p \in \Lambda$, denote

$$
a_{p}=\operatorname{deg}_{p}(n), \quad b_{p}=\operatorname{deg}_{p}(m), \quad c_{p}=\operatorname{deg}_{p}(t), \quad d_{p}=\operatorname{deg}_{p}(q) .
$$

Subdivide $\Lambda$ as $\Lambda=\Lambda_{1} \sqcup \Lambda_{2} \sqcup \Lambda^{\prime}$, with

$$
\Lambda_{1}=\left\{p: d_{p}>0\right\}, \quad \Lambda_{2}=\left\{p: a_{p}>0, d_{p}=0\right\}, \quad \Lambda^{\prime}=\left\{p: b_{p}>0, a_{p}=0\right\} .
$$

Denote

$$
e=\operatorname{deg}_{2}(r+1)
$$

It follows from $t \mid n$ and $t(r-1) \equiv 0(\bmod n)$ that

$$
\begin{cases}a_{p}-d_{p} \leq c_{p} \leq a_{p}, & p \in \Lambda_{1} \\ c_{p}=a_{p}, & p \in \Lambda_{2}\end{cases}
$$

and it follows from $r^{m}-1 \equiv 0(\bmod n)$ and Lemma 2.1 (II) that

$$
d_{p}+b_{p} \geq a_{p}, \quad \text { for all } p \in \Lambda_{1} \text { with }\left(p, d_{p}\right) \neq(2,1) \text { or }\left(p, d_{p}, b_{p}\right)=(2,1,0) \text {; }
$$

finally, when $d_{2}=1$ and $b_{2}>0$, Lemma 2.1 (II) applied to $r^{m}-1=\left(r^{2}\right)^{m / 2}-1$ implies

$$
e+b_{2} \geq a_{2}
$$

The condition (8) is equivalent to

$$
\min \left\{d_{p}, c_{p}\right\}+\operatorname{deg}_{p}\left(y_{1}\right) \geq b_{p} \quad \text { for all } \quad p \in \Lambda .
$$

Suppose that $x_{1}, x_{2}, y_{1}, y_{2}$ satisfy the conditions (8), (9) and (10) and let $\sigma$ be the endomorphism of $H$ given in Lemma 2.2. Since $H$ is finite, $\sigma$ is invertible if and only if it is injective, which is equivalent to that both the induced homomorphism $\bar{\sigma}: H^{\mathrm{ab}} \rightarrow H^{\mathrm{ab}}$ and the restriction $\sigma_{0}:=\left.\sigma\right|_{[H, H]}$ are injective.

In the remainder of this subsection, let

$$
w=\frac{t y_{1}}{m} .
$$

Lemma 2.3. The homomorphism $\bar{\sigma}$ is injective if and only if

$$
\begin{cases}p \nmid y_{2}, & p \in \Lambda^{\prime}, \\ p \nmid x_{1}+w, & p \in \Lambda_{1} \text { with } b_{p} c_{p}=0, \\ p \nmid x_{1} y_{2}-x_{2} y_{1}, & p \in \Lambda_{1} \text { with } b_{p}, c_{p}>0 .\end{cases}
$$


Proof. For each $p \in \Lambda^{\prime} \sqcup \Lambda_{1}$, let

$$
H_{p}^{\mathrm{ab}}=\left\langle\bar{\alpha}_{p}, \bar{\beta}_{p}\right\rangle, \quad \text { with } \quad \bar{\alpha}_{p}=\frac{t q}{p^{c_{p}+d_{p}}} \bar{\alpha}, \quad \bar{\beta}_{p}=\frac{m q}{p^{b_{p}+d_{p}}} \bar{\beta} ;
$$

it is the Sylow $p$-subgroup of $H^{\mathrm{ab}}$. Then $\bar{\sigma}$ is injective if and only if $\bar{\sigma}_{p}:=\left.\bar{\sigma}\right|_{H_{p}^{\mathrm{ab}}}$ is injective for all $p$. Take an integer $z_{p}$ with $\left(t / p^{c_{p}}\right) z_{p} \equiv 1\left(\bmod p^{d_{p}}\right)$. We have

$$
\begin{aligned}
& \bar{\sigma}_{p}\left(\bar{\alpha}_{p}\right)=\frac{t q}{p^{c_{p}+d_{p}}}\left(x_{1} \bar{\alpha}+y_{1} \bar{\beta}\right)=x_{1} \bar{\alpha}_{p}+\frac{p^{b_{p}} t y_{1}}{p^{c_{p}} m} \bar{\beta}_{p}, \\
& \bar{\sigma}_{p}\left(\bar{\beta}_{p}\right)=\frac{m q}{p^{b_{p}+d_{p}}}\left(x_{2} \bar{\alpha}+y_{2} \bar{\beta}\right)=\frac{m}{p^{b_{p}}} z_{p} x_{2} \bar{\alpha}_{p}+y_{2} \bar{\beta}_{p} .
\end{aligned}
$$

Let $\check{H}_{p}=H_{p}^{\mathrm{ab}} / p H_{p}^{\mathrm{ab}}$, let $\check{\alpha}_{p}, \check{\beta}_{p}$ denote the images of $\bar{\alpha}_{p}, \bar{\beta}_{p}$ under the quotient homomorphism $H_{p}^{\mathrm{ab}} \rightarrow \check{H}_{p}$, and let $\check{\sigma}_{p}$ denote the endomorphism of $\check{H}_{p}$ induced from $\bar{\sigma}_{p}$. Then $\bar{\sigma}_{p}$ is injective if and only if $\check{\sigma}_{p}$ is injective. It follows from (23), (24) that

$$
\begin{aligned}
& \check{\sigma}_{p}\left(\check{\alpha}_{p}\right)=x_{1} \check{\alpha}_{p}+\frac{p^{b_{p}} t y_{1}}{p^{c_{p}} m} \check{\beta}_{p}, \\
& \check{\sigma}_{p}\left(\check{\beta}_{p}\right)=\frac{m}{p^{b_{p}}} z_{p} x_{2} \check{\alpha}_{p}+y_{2} \check{\beta}_{p} .
\end{aligned}
$$

- If $b_{p}>d_{p}=0$, then $\check{\alpha}_{p}=0, \check{H}_{p}=\left\langle\check{\beta}_{p}\right\rangle \cong \mathbb{Z}_{p}$, and by (26),$\check{\sigma}_{p}$ is injective if and only if $p \nmid y_{2}$.

- If $d_{p}>b_{p}=0$, then $\check{\beta}_{p}=p^{c_{p}} \check{\alpha}_{p}, \check{H}_{p}=\left\langle\check{\alpha}_{p}\right\rangle \cong \mathbb{Z}_{p}$, and by (25), $\check{\sigma}_{p}$ is injective if and only if $p \nmid x_{1}+w$.

- If $d_{p}>c_{p}=0$, then $\check{\alpha}_{p}=p^{b_{p}} \check{\beta}_{p}, \check{H}_{p}=\left\langle\check{\beta}_{p}\right\rangle$, and by (26), $\check{\sigma}_{p}$ is injective if and only if $p \nmid m z_{p} x_{2}+y_{2}$, which, by (9), is equivalent to $p \nmid x_{1}+w$.

- If $b_{p}, c_{p}, d_{p}>0$, then $\check{H}_{p}=\left\langle\check{\alpha}_{p}, \check{\beta}_{p}\right\rangle \cong \mathbb{Z}_{p}^{2}$, and by (25), (26), $\bar{\sigma}_{p}$ is invertible if and only if

$$
0 \not \equiv x_{1} y_{2}-\frac{p^{b_{p}} t y_{1}}{p^{c_{p}} m} \cdot \frac{m}{p^{b_{p}}} z_{p} x_{2} \equiv x_{1} y_{2}-x_{2} y_{1} \quad(\bmod p) .
$$

Lemma 2.4. Suppose $p \nmid x_{1} y_{2}-x_{2} y_{1}$ for all $p \in \Lambda_{1}$ with $d_{p}<a_{p}$. Then the homomorphism $\sigma_{0}$ is injective if and only if

$$
r^{y_{1}} \equiv 1 \quad\left(\bmod p^{a_{p}}\right) \quad \text { and } \quad p \nmid x_{1}+w \quad \text { for all } \quad p \in \Lambda_{2} .
$$

Proof. Note that $\sigma_{0}\left(\alpha^{r-1}\right)=\alpha^{u}$, with

$$
u=x_{1}[r-1]_{r y_{1}}+(r-1) w .
$$


For each $p \in \Lambda_{1}$ with $d_{p}<a_{p}$, by (10) we have

$$
\begin{aligned}
u & \equiv\left(1-r^{y_{1}}\right) x_{1}[r-1]_{r^{y_{1}}}+x_{1}\left(r^{y_{2}}-1\right)-x_{2}\left(r^{y_{1}}-1\right) \quad\left(\bmod p^{a_{p}}\right) \\
& \equiv(r-1)\left(x_{1} y_{2}-x_{2} y_{1}\right) \quad\left(\bmod p^{d_{p}+1}\right),
\end{aligned}
$$

the second line following from $r^{y_{j}}-1 \equiv(r-1) y_{j}\left(\bmod p^{2 d_{p}}\right), j=1,2$. Hence

$$
\operatorname{deg}_{p}(u)=d_{p}
$$

Thus $\sigma_{0}$ is injective if and only if $p \nmid u$ for all $p \in \Lambda_{2}$. For $p \in \Lambda_{2}$, by (17), (20),

$$
\operatorname{deg}_{p}(w)=c_{p}+\operatorname{deg}_{p}\left(y_{1}\right)-b_{p} \geq c_{p}=a_{p} .
$$

Hence, if $p \nmid u$ then $p \nmid x_{1}[r-1]_{r^{y_{1}}}$ and this implies that $r^{y_{1}} \equiv 1\left(\bmod p^{a_{p}}\right)$ (by the argument given). On the other hand, if $r^{y_{1}} \equiv 1(\bmod p)$ then $[r-1]_{r^{y_{1}}} \equiv r-1 \not \equiv 0\left(\bmod p^{a_{p}}\right)$ and hence $p \mid u$ if and only if $p \mid x_{1}$. Therefore, $\sigma_{0}$ is injective if and only if $p \nmid u$ if and only if $r^{y_{1}} \equiv 1$ $\left(\bmod p^{a_{p}}\right)$ and $p \nmid x_{1}$; the condition $p \nmid x_{1}$ is equivalent to $p \nmid x_{1}+w$.

Remark 2.5. In order to obtain neat conditions, we prefer $p \nmid x_{1}+w$ to $p \nmid x_{1}$.

Summarizing, sufficient and necessary conditions for $\sigma$ to be an automorphism are (8), (9), (10), (22) and (27). Let (9) $p$ (resp. (10) $p$ ) denote the condition (9) (resp. (10)) with mod $n$ replaced by $\bmod p^{a_{p}}$. Then (9) (resp. (10)) is equivalent to (9) $p\left(\right.$ resp. (10) $p$ ) for all $p \in \Lambda_{1} \sqcup \Lambda_{2}$ simultaneously.

Remark 2.6. If $p \in \Lambda_{2}$, then $p \neq 2$ : otherwise $2 \mid n$ but $2 \nmid r-1$, contradicting $n \mid r^{m}-1$. Due to (17), (27), the conditions (91) $p,(10) p$ are equivalent to $r^{y_{2}-1} \equiv 1\left(\bmod p^{a_{p}}\right)$.

If $p \in \Lambda_{1}$ with $d_{p}=a_{p}$, then $r \equiv 1\left(\bmod p^{a_{p}}\right)$, hence (10) $p$ is trivial, and (9) $)_{p}$ becomes $t\left(x_{1}+w-y_{2}\right) \equiv m x_{2}\left(\bmod p^{a_{p}}\right)$.

Suppose $p \in \Lambda_{1}$ with $d_{p}<a_{p}$. Note that by (18), $b_{p}>0$. We shall simplify (91) $p$ and (10) $p$, with (8) and (22) assumed.

By Lemma 2.1 (I), $[r-1]_{r^{y_{1}}} \equiv r-1\left(\bmod p^{2 d_{p}}\right)$ when $p \neq 2$ or $p=2, \operatorname{deg}_{2}\left(r^{y_{1}}-1\right)>1$. Hence by (29),

$$
p \nmid x_{1}+w \quad \text { if } \quad p \neq 2 \text { or } \quad p=2, d_{2}+\operatorname{deg}_{2}\left(y_{1}\right)>1 .
$$

By (17), (18), (20),

$$
\begin{aligned}
\operatorname{deg}_{p}\left(y_{1}\right) \geq b_{p}-d_{p} \geq a_{p}-2 d_{p}, & \text { if } \quad\left(p, d_{p}\right) \neq(2,1), \\
\operatorname{deg}_{p}(w)=\operatorname{deg}_{p}\left(y_{1}\right)+c_{p}-b_{p} \geq c_{p}-d_{p} \geq a_{p}-2 d_{p}, & \text { if } \quad\left(p, d_{p}\right) \neq(2,1) .
\end{aligned}
$$

We will use (30), (31), (32) repeatedly.

Lemma 2.7. If $2 \neq p \in \Lambda_{1}$, then the conditions (91) $p$ and (10) $p$ hold if and only if

$$
\begin{aligned}
m x_{2} & \equiv t\left(x_{1}+w-y_{2}\right) \quad\left(\bmod p^{a_{p}}\right), \\
y_{2} & \equiv 1+w \quad\left(\bmod p^{a_{p}-d_{p}}\right) .
\end{aligned}
$$


Proof. Abbreviate $a_{p}, b_{p}, c_{p}, d_{p}, \operatorname{deg}_{p}(x)$ to $a, b, c, d, \operatorname{deg}(x)$, respectively.

Applying Lemma 2.1, with (17), (18), (31) recalled, we obtain

$$
\begin{aligned}
r^{y_{1}} \equiv 1+(r-1) y_{1}, \quad[t]_{r^{y_{1}}} \equiv t, \quad[m]_{r} y_{2} \equiv m & \left(\bmod p^{a}\right), \\
{[r]_{r^{y_{1}}}=\left(r^{y_{1}}\right)^{r-1}+[r-1]_{r^{y_{1}}} \equiv 1+(r-1)=r } & \left(\bmod p^{a}\right) .
\end{aligned}
$$

Hence $(9)_{p}$ can be simplified as (33) and $(10) p$ can be rewritten as

$$
(r-1) y_{1} x_{2}+(r-1) w \equiv\left(r^{y_{2}}-r\right) x_{1} \quad\left(\bmod p^{a}\right) .
$$

By (31) and (32), $\operatorname{deg}\left((r-1) y_{1} x_{2}+(r-1) w\right) \geq a-d$, hence

$$
\operatorname{deg}\left(y_{2}-1\right)+\operatorname{deg}\left(x_{1}\right)=\operatorname{deg}\left(\left(r^{y_{2}}-r\right) x_{1}\right)-d \geq a-2 d .
$$

By Lemma $2.1(\mathrm{II}), r^{y_{2}-1}-1 \equiv(r-1)\left(y_{2}-1\right)\left(\bmod p^{a-\operatorname{deg}\left(x_{1}\right)}\right)$, and then

$$
\left(r^{y_{2}}-r\right) x_{1}=(r-1)^{2}\left(y_{2}-1\right) x_{1}+(r-1)\left(y_{2}-1\right) x_{1} \equiv(r-1)\left(y_{2}-1\right) x_{1} \quad\left(\bmod p^{a}\right) .
$$

Thus (35) can be converted into $\left(y_{2}-1\right) x_{1} \equiv y_{1} x_{2}+w\left(\bmod p^{a-d}\right)$. Since by (33),

$$
\begin{aligned}
y_{1} x_{2} & \equiv \frac{t y_{1}}{m}\left(x_{1}+w-y_{2}\right)=w\left(x_{1}+w-y_{2}\right) \quad\left(\bmod p^{a+\operatorname{deg}\left(y_{1}\right)-b}\right) \\
& \equiv w\left(x_{1}+w-y_{2}\right) \quad\left(\bmod p^{a-d}\right),
\end{aligned}
$$

we are led to $\left(y_{2}-1\right) x_{1} \equiv w\left(x_{1}+w-y_{2}+1\right)\left(\bmod p^{a-d}\right)$, i.e.,

$$
\left(y_{2}-1-w\right)\left(x_{1}+w\right) \equiv 0 \quad\left(\bmod p^{a-d}\right) ;
$$

due to (30), this is equivalent to (34).

Set

$$
f\left(y_{1}\right)= \begin{cases}2^{a_{2}-d_{2}-1}, & \text { if } c_{2} \neq b_{2}, \min \left\{b_{2}, c_{2}\right\}=a_{2}-d_{2} \text { and } \operatorname{deg}_{2}\left(y_{1}\right)=b_{2}-d_{2}, \\ 0, & \text { otherwise. }\end{cases}
$$

Lemma 2.8. If $2 \in \Lambda_{1}$, then the conditions $(\sqrt{9})_{2}$ and $(\sqrt{10})_{2}$ hold if and only if

(i) if $b_{2}=c_{2}=d_{2}=1$ (so that $a_{2}=2$ ), then no additional condition is required;

(ii) if $d_{2}=1$ and $\max \left\{b_{2}, c_{2}\right\}>1$, then $2 \mid y_{1}, \operatorname{deg}_{2}\left(x_{2}\right) \geq a_{2}-b_{2}-e+1$ and

$$
w \equiv 2^{e-1}\left(y_{1}-y_{2}+1\right) \quad\left(\bmod 2^{a_{2}-1}\right) ;
$$

(iii) if $d_{2}>1$, then

$$
\begin{aligned}
m x_{2} & \equiv t\left(x_{1}+w-y_{2}\right) \quad\left(\bmod 2^{a_{2}}\right), \\
y_{2} & \equiv 1+w+f\left(y_{1}\right) \quad\left(\bmod 2^{a_{2}-d_{2}}\right) .
\end{aligned}
$$


Proof. Abbreviate $a_{2}, b_{2}, c_{2}, d_{2}, \operatorname{deg}_{2}(x)$ to $a, b, c, d, \operatorname{deg}(x)$, respectively.

(i) For any $x, u>0$, we have $r^{x} \equiv 1+2 x(\bmod 4)$, and

$$
[u]_{r^{x}}=\sum_{i=0}^{u-1} r^{i x} \equiv \sum_{i=0}^{u-1}(1+2 i x) \equiv u+u(u-1) x \quad(\bmod 4) .
$$

In particular, $[m]_{r^{y_{2}}} \equiv 2+2 y_{2},[t]_{r^{y_{1}}} \equiv 2+2 y_{1},[r]_{r^{y_{1}}} \equiv 3+2 y_{1}(\bmod 4)$. The condition (9) 2 , (10) 2 can be converted into, respectively,

$$
\begin{array}{rlr}
\left(x_{2}+1\right)\left(y_{2}+1\right)-\left(x_{1}+1\right)\left(y_{1}+1\right) & \equiv 0 & (\bmod 2), \\
x_{2} y_{1}+x_{1}\left(1+y_{1}-y_{2}\right)+y_{1} & \equiv 0 & (\bmod 2) .
\end{array}
$$

Due to (22),$x_{2} y_{1} \equiv x_{1} y_{2}+1(\bmod 2)$, hence (44) is equivalent to $\left(x_{1}+1\right)\left(y_{1}+1\right) \equiv 0(\bmod 2)$, which is true since by (22), at least one of $x_{1}, y_{1}$ is odd. Then similarly, (43) also holds.

(ii) We first show $2 \mid y_{1}$. Assume on the contrary that $2 \nmid y_{1}$. By (20), $b=1$, so that $c>1$. By $(9)_{2}, x_{2}[m]_{r^{y_{2}}} \equiv 0(\bmod 4)$, which forces $2 \nmid y_{2}$ : if $2 \mid y_{2}$, then $r^{y_{2}} \equiv 1(\bmod 4)$, so that $4 \nmid[m]_{r} y_{2}$, we would get $2 \mid x_{2}$, contradicting (22). Then $r^{y_{j}} \equiv-1(\bmod 4), j=1,2$, and $[r]_{r^{y_{1}}} \equiv 1(\bmod 4)$, so (10) 2 implies $2\left(x_{1}-x_{2}\right) \equiv 0(\bmod 4)$. But this contradicts (22) .

Thus $2 \mid y_{1}$. By (22), $2 \nmid x_{1} y_{2} ;$ by (30), $2 \mid w$. Hence

$$
t\left(x_{1}+w-y_{2}\right) \equiv 0 \quad\left(\bmod 2^{a}\right) .
$$

By (19), (20), $1+\operatorname{deg}\left(y_{1}\right)+e \geq b+e \geq a$, hence

$$
\operatorname{deg}\left(r^{y_{1}}-1\right)=\operatorname{deg}\left(\left(r^{2}\right)^{y_{1} / 2}-1\right)=e+\operatorname{deg}\left(y_{1}\right) \geq a-1 .
$$

When $c>1$, applying Lemma 2.1, we obtain

$$
\begin{aligned}
{[t]_{r^{y_{1}}} } & \equiv\left(1+2^{e+\operatorname{deg}\left(y_{1}\right)-1}\right) t \quad\left(\bmod 2^{e+\operatorname{deg}\left(y_{1}\right)+c}\right) \\
& \equiv t \quad\left(\bmod 2^{a}\right), \\
{[r]_{r^{y_{1}}} } & =\left(r^{y_{1}}\right)^{r-1}+[r-1]_{r^{y_{1}}} \equiv 1+\left(1+2^{e+\operatorname{deg}\left(y_{1}\right)-1}\right)(r-1) \quad\left(\bmod 2^{e+\operatorname{deg}\left(y_{1}\right)+1}\right) \\
& \equiv r+2^{e} y_{1} \quad\left(\bmod 2^{a}\right) ;
\end{aligned}
$$

when $c=1$ so that $a=2$, these congruence relations obviously hold.

Due to (45), the condition $(9)_{2}$ becomes $x_{2}[m]_{r y_{2}} \equiv 0\left(\bmod 2^{a}\right)$. Since $\operatorname{deg}\left(r^{y_{2}}+1\right)=$ $\operatorname{deg}(r+1)=e$ and $[m]_{r^{y_{2}}}=\left(r^{y_{2}}+1\right)[m / 2]_{r^{2 y_{2}}}$, we have $\operatorname{deg}\left([m]_{r^{y_{2}}}\right)=e+b-1$. Hence

$$
\operatorname{deg}\left(x_{2}\right) \geq a-b-e+1 \text {. }
$$

This together with (20) implies

$$
\operatorname{deg}\left(\left(r^{y_{1}}-1\right) x_{2}\right)=\operatorname{deg}\left(y_{1}\right)+e+\operatorname{deg}\left(x_{2}\right) \geq b-1+e+\operatorname{deg}\left(x_{2}\right) \geq a .
$$

Then $(10)_{2}$ becomes

$$
x_{1}\left(r^{y_{2}}-r-2^{e} y_{1}\right) \equiv(r-1) w \quad\left(\bmod 2^{a}\right) .
$$


Since $\operatorname{deg}\left(r^{y_{2}-1}-1\right)=\operatorname{deg}\left(\left(r^{2}\right)^{\left(y_{2}-1\right) / 2}-1\right)=e+\operatorname{deg}\left(y_{2}-1\right)$, we have $r^{y_{2}-1}-1=2^{e}\left(y_{2}-1\right) z$ for some odd $z$. Using $2^{e+1} y_{1} \equiv 2(r-1) w \equiv 0\left(\bmod 2^{a}\right)$, we can convert (47) into (40).

(iii) Applying Lemma 2.1 (with (31) recalled), we obtain

$$
\begin{aligned}
r^{y_{1}} & \equiv\left\{\begin{array}{ll}
1+(r-1) y_{1}, & 2 \nmid y_{1}, \\
1+\left(r-1+2^{2 d-1}\right) y_{1}, & 2 \mid y_{1}
\end{array} \quad\left(\bmod 2^{a}\right),\right. \\
{[r]_{r^{y_{1}}} } & \equiv r+2^{2 d-1} y_{1} \quad\left(\bmod 2^{a}\right), \\
{[t]_{r^{y_{1}}} } & \equiv\left(1+2^{d-1} y_{1}\right) t \quad\left(\bmod 2^{a}\right), \\
{[m]_{r^{y_{2}}} } & \equiv\left(1+2^{d-1} y_{2}\right) m \quad\left(\bmod 2^{a}\right) .
\end{aligned}
$$

We deal with the cases $2 \mid y_{1}$ and $2 \nmid y_{1}$ separately.

(iii-1) If $2 \mid y_{1}$, then by (22), $2 \nmid x_{1} y_{2}$, and by (30), $2 \mid w$. The condition (9) 2 becomes

$$
\left(1+2^{d-1} y_{2}\right) m x_{2} \equiv t\left(x_{1}+w-y_{2}\right) \quad\left(\bmod 2^{a}\right),
$$

which can be converted into (41) via multiplying $1-2^{d-1} y_{2}$. Moreover, (48) implies $b+\operatorname{deg}\left(x_{2}\right) \geq$ $\min \{c+1, a\}$, hence

$$
\begin{aligned}
2 d-1+\operatorname{deg}\left(x_{2}\right)+\operatorname{deg}\left(y_{1}\right) & \geq 2 d-1+(\min \{c+1, a\}-b)+(b-d) \\
& =d-1+\min \{c+1, a\} \geq a .
\end{aligned}
$$

As a result, $x_{2}\left(r^{y_{1}}-1\right) \equiv(r-1) x_{2} y_{1}\left(\bmod 2^{a}\right)$. Using this and $2^{2 d-1}\left(x_{1}-1\right) y_{1} \equiv 0\left(\bmod 2^{a}\right)$, we may convert $(10)_{2}$ into

$$
(r-1) x_{2} y_{1}+2^{2 d-1} y_{1}+\left(r-r^{y_{2}}\right) x_{1}+(r-1) w \equiv 0 \quad\left(\bmod 2^{a}\right) .
$$

By an argument similar as that deducing (36) in the proof of Lemma 2.7, we obtain $\operatorname{deg}\left(y_{2}-1\right) \geq$ $a-2 d$, and then by Lemma 2.1 (II),

$$
r^{y_{2}-1}-1 \equiv\left(1+2^{d-1}\right)(r-1)\left(y_{2}-1\right) \quad\left(\bmod 2^{a}\right) .
$$

Using $(r-1)\left(r^{y_{2}-1}-1\right) \equiv 0\left(\bmod 2^{a}\right)$, we can further convert (49) into

$$
\left(y_{2}-1\right) x_{1} \equiv y_{1} x_{2}+w+2^{d-1}\left(y_{1}-y_{2}+1\right) \quad\left(\bmod 2^{a-d}\right) .
$$

Similarly as (37), it follows from (41) that $y_{1} x_{2} \equiv w\left(x_{1}+w-y_{2}\right)\left(\bmod 2^{a-d}\right)$, and then (50) becomes

$$
\left(y_{2}-1-w\right)\left(x_{1}+w+2^{d-1}\right) \equiv 2^{d-1}\left(y_{1}-w\right) \quad\left(\bmod 2^{a-d}\right) .
$$

From (31) and (32) we see that $\operatorname{deg}\left(y_{1}-w\right) \geq a-2 d$, and the equality holds if and only if one of the following cases occurs:

- $\operatorname{deg}(w)>\operatorname{deg}\left(y_{1}\right)=a-2 d$, which is equivalent to $\operatorname{deg}\left(y_{1}\right)=b-d$ and $c>b=a-d$; 
- $\operatorname{deg}\left(y_{1}\right)>\operatorname{deg}(w)=a-2 d$, which is equivalent to $\operatorname{deg}\left(y_{1}\right)=b-d$ and $b>c=a-d$.

Thus (51) becomes

$$
\left(y_{2}-1-w\right)\left(x_{1}+w+2^{d-1}\right) \equiv f\left(y_{1}\right) \equiv f\left(y_{1}\right)\left(x_{1}+w+2^{d-1}\right) \quad\left(\bmod 2^{a-d}\right),
$$

which is equivalent to (42).

(iii-2) If $2 \nmid y_{1}$, then $d, c \geq b$, and $2 d \geq a$. By Lemma 2.1(II), $r^{y_{2}} \equiv 1+(r-1) y_{2}\left(\bmod 2^{a}\right)$, hence (91) 2 , (10) 2 become, respectively,

$$
\begin{aligned}
& \left(1+2^{d-1} y_{2}\right) m x_{2}+t y_{2} \equiv\left(1+2^{d-1}\right) t x_{1}+t w \quad\left(\bmod 2^{a}\right), \\
& \left(y_{2}-1\right) x_{1} \equiv y_{1} x_{2}+w+2^{d-1} x_{1} \quad\left(\bmod 2^{a-d}\right) .
\end{aligned}
$$

If $c=b$, then by (30), $2 \mid x_{1}$, and by (22), $2 \nmid x_{2}$. By (52), $2 \mid y_{2}$, and then (52) becomes (41). We can reduce (53) to $y_{2}-1 \equiv w\left(\bmod 2^{a-d}\right)$ similarly as in the proof of Lemma 2.7 ,

Now assume $c>b$ so that $2 \mid w$. By (30), $2 \nmid x_{1}$. Since $c+d-1 \geq b+d \geq a$, we can reduce (52) to (41) via multiplying by $1-2^{d-1} y_{2}$. If $2 d>a$, then still similarly as in the proof of Lemma 2.7, we can reduce (53) to $y_{2}-1 \equiv w\left(\bmod 2^{a-d}\right)$; if $2 d=a$, then $b=a-d=d$, then similarly as in (iii-1), we can reduce (53) to $y_{2}-1 \equiv w+2^{a-d-1}\left(\bmod 2^{a-d}\right)$.

Thus in any case, $(91)_{2},(101)_{2}$ are equivalent to (41), (42).

\subsection{Main result}

Let $m_{0}$ be the smallest positive integer $k$ such that $r^{k} \equiv 1\left(\bmod p^{a_{p}}\right)$ for all $p \in \Lambda_{2}$. Combining Lemma 2.3, Lemma 2.4, Remark 2.6, Lemma 2.7 and Lemma 2.8, we establish

Theorem 2.9. Each automorphism of $H(n, m ; t, r)$ is given by

$$
\alpha^{u} \beta^{v} \mapsto \exp _{\alpha}\left(x_{1}[u]_{r} y_{1}+r^{y_{1} u} x_{2}[v]_{r y_{2}}\right) \beta^{y_{1} u+y_{2} v}, \quad u, v>0
$$

for a unique quadruple $\left(x_{1}, x_{2}, y_{1}, y_{2}\right)$ with $0<x_{1}, x_{2} \leq n, 0<y_{1}, y_{2} \leq m$ and such that

(i) for all $p \in \Lambda$,

$$
\begin{cases}p \nmid y_{2}, & p \in \Lambda^{\prime}, \\ p \nmid x_{1}+t y_{1} / m, & p \in \Lambda_{2} \text { or } p \in \Lambda_{1} \text { with } b_{p} c_{p}=0, \\ p \nmid x_{1} y_{2}-x_{2} y_{1}, & p \in \Lambda_{1} \text { with } b_{p}, c_{p}>0 ;\end{cases}
$$

(ii) $(r-1, t) y_{1} \equiv 0(\bmod m)$ and $y_{1} \equiv y_{2}-1 \equiv 0\left(\bmod m_{0}\right)$;

(iii) for all $p \in \Lambda_{1}$ with $p \neq 2$ or $p=2, a_{2}=d_{2}$,

$$
\begin{aligned}
m x_{2} & \equiv t\left(x_{1}+t y_{1} / m-y_{2}\right) \quad\left(\bmod p^{a_{p}}\right), \\
y_{2} & \equiv 1+t y_{1} / m \quad\left(\bmod p^{a_{p}-d_{p}}\right) ;
\end{aligned}
$$


(iv) if $\max \left\{b_{2}, c_{2}\right\}>d_{2}=1$ and $a_{2}>1$, then $2 \mid y_{1}, \operatorname{deg}_{2}\left(x_{2}\right) \geq a_{2}-b_{2}-e+1$ and

$$
t y_{1} / m \equiv 2^{e-1}\left(y_{1}-y_{2}+1\right) \quad\left(\bmod 2^{a_{2}-1}\right) ;
$$

(v) if $d_{2}>1$, then

$$
\begin{aligned}
m x_{2} & \equiv t\left(x_{1}+t y_{1} / m-y_{2}\right) \quad\left(\bmod 2^{a_{2}}\right), \\
y_{2} & \equiv 1+t y_{1} / m+f\left(y_{1}\right) \quad\left(\bmod 2^{a_{2}-d_{2}}\right) .
\end{aligned}
$$

\section{References}

[1] J.N.S. Bidwell, M.J. Curran, The automorphism group of a split metacyclic p-group. Arch. Math. 87 (2006), 488-497.

[2] H.-M. Chen, Reduction and regular t-balanced Cayley maps on split metacyclic 2-groups. arXiv:1702.08351.

[3] M.J. Curran, The automorphism group of a split metacyclic 2-group. Arch. Math. 89 (2007), $10-23$.

[4] M.J. Curran, The automorphism group of a nonsplit metacyclic p-group. Arch. Math. 90 (2008), 483-489.

[5] R.M. Davitt, The automorphism group of a finite metacyclic p-group. Proc. Amer. Math. Soc. 25 (1970), 876-879.

[6] M. Golasiński, D.L. Gonçalves, On automorphisms of split metacyclic groups. Manuscripta Math. 128 (2009), 251-273.

[7] C.E. Hempel, Metacyclic groups. Commun. Algebra 28 (2000), no. 8, 3865-3897.

[8] H.J. Zassenhaus, The theory of groups. Second edition, Chelsea, New York, 1956. 